\title{
Do Nuclear Star Clusters and Supermassive Black Holes Follow the Same Host-Galaxy Correlations?
}

\author{
Peter Erwin $^{1,2}$ and Dimitri Alexei Gadotti ${ }^{3}$ \\ ${ }^{1}$ Max-Planck-Institut für Extraterrestrische Physik, Giessenbachstraße, 85748 Garching, Germany \\ ${ }^{2}$ Universitäts-Sternwarte München, Scheinerstraße 1, 81679 München, Germany \\ ${ }^{3}$ European Southern Observatory, Alonso de Cordova 3107, Vitacura, Casilla 19001, Santiago 19, Chile \\ Correspondence should be addressed to Peter Erwin, erwin@mpe.mpg.de \\ Received 6 October 2011; Revised 1 December 2011; Accepted 6 December 2011 \\ Academic Editor: Isabelle Gavignaud
}

Copyright ( $) 2012$ P. Erwin and D. A. Gadotti. This is an open access article distributed under the Creative Commons Attribution License, which permits unrestricted use, distribution, and reproduction in any medium, provided the original work is properly cited.

Studies have suggested that there is a strong correlation between the masses of nuclear star clusters (NSCs) and their host galaxies, a correlation which is said to be an extension of the well-known correlations between supermassive black holes (SMBHs) and their host galaxies. But careful analysis of disk galaxies_including 2D bulge/disk/bar decompositions_shows that while SMBHs correlate with the stellar mass of the bulge component of galaxies, the masses of NSCs correlate much better with the total galaxy stellar mass. In addition, the mass ratio $M_{\mathrm{NSC}} / M_{\star \text {, tot }}$ for NSCs in spirals (at least those with Hubble types Sc and later) is typically an order of magnitude smaller than the mass ratio $M_{\mathrm{BH}} / M_{\star, \text { bul }}$ of SMBHs. The absence of a universal "central massive object" correlation argues against common formation and growth mechanisms for both SMBHs and NSCs. We also discuss evidence for a break in the NSC-host galaxy correlation, galaxies with Hubble types earlier than Sbc appear to host systematically more massive NSCs than do types Sc and later.

\section{Introduction}

As far as we can tell, all massive galaxies in the local universe harbor supermassive black holes (SMBHs, with masses $\left.M_{\mathrm{BH}} \sim 10^{6}-10^{9} M_{\odot}\right)$. The masses of these SMBHs correlate strongly with several global properties of the host galaxies, particularly with the central velocity dispersion $\sigma_{0}[1,2]$ and with the bulge luminosity or mass (e.g., [3, 4]). These correlations imply that the processes which drive galaxy growth and the processes which drive black hole growth are intimately linked-perhaps even the same processes.

It is now also clear that many galaxies, particularly latertype spirals, host luminous nuclear star clusters (NSCs; e.g., $[5,6])$, with masses in the range $10^{5}-10^{8} M_{\odot}$; see the review by Böker [7] for more details. Recently, several authors have argued that NSCs and central SMBHs have the same hostgalaxy correlations, in particular, that SMBHs and NSCs have the same correlation with bulge luminosity and mass [8-11] (but see Balcells et al. [12]). The suggestion, then, is that
NSCs and SMBHs are in a sense members of the same family of "Central Massive Objects" (CMOs), and thus that they may have grown via the same mechanisms (e.g., [13-16]).

We argue, however, that one should be cautious about assuming that NSCs and SMBHs are really part of the same family, with the same host-galaxy relationships. To begin with, the samples of Wehner and Harris [8] and Ferrarese et al. [9], which were used to make the CMO argument, were almost entirely early-type galaxies-mostly ellipticals and dwarf ellipticals. These are galaxies which are, in essence, "pure bulge" systems, so one could just as easily argue for a correlation with total galaxy mass. But we know that SMBHs in disk galaxies correlate better with just the bulge, and not with the total galaxy mass or light (e.g., [17, 18]). Given that there have been previous claims that NSCs in spiral galaxies correlate with the total galaxy light (e.g., [19]), we are prompted ask the question: do nuclear clusters in disk galaxies correlate with the bulge (like SMBHs), or with the whole galaxy? 


\section{Samples, Methodology, and Data Sources}

Although current studies suggest that the $M_{\mathrm{BH}}-\sigma_{0}$ relation is tighter and has less intrinsic scatter than the $M_{\mathrm{BH}}-M_{\star \text {,bul }}$ relation (e.g., [20]), velocity dispersion is not the ideal hostgalaxy measure to use here, for three reasons. First, most of the best-determined NSC masses are based directly on the measured velocity dispersion of the NSC (e.g., [21]), which is often indistinguishable from that of the surrounding bulge; this means a (spurious) correlation between NSC mass, and central velocity dispersion is only to be expected. Second, some NSCs are found in galaxies with no detectable bulge at all (see discussion in Section 3). Finally, it is difficult to see how one should discriminate between a velocity dispersion due to the bulge versus one due to the whole galaxy. But discriminating between bulge and whole-galaxy luminosities and masses is much simpler. So we choose instead to compare NSCs and their host galaxies with the $M_{\mathrm{BH}}-M_{\star, \text { bul }}$ relation, which means comparing NSC masses with the stellar masses of host galaxies and their bulges.

For NSCs, we emphasize galaxies where the NSC masses have been dynamically measured, since this is the most direct analog to well-determined SMBH masses (i.e., those with direct dynamical mass measurements from stellar, gas, or maser kinematics, where the SMBH sphere of influence is resolved). In addition, dynamical measurements avoid possible problems with multiple stellar populations; the latter can potentially bias stellar masses estimated from broadband colors. Spectroscopic studies [11,22] have shown that NSCs often contain multiple stellar populations; this renders mass estimates based on single stellar population (SSP) models (e.g., those used by [9]) somewhat uncertain. The NSCs we focus on are taken primarily from the sample of Walcher et al. [21], with additional data from Ho and Filippenko [23], Böker et al. [24], Kormendy and Bender [25], Matthews et al. [26] and Gebhardt et al. [27], Barth et al. [28], Seth et al. [29], and Kormendy et al. [30]; we use the estimate of Launhardt et al. [31] for the Milky Way's NSC. This gives us a total of 18 galaxies with dynamically determined NSC masses. These cover Hubble types S0-Sm, but the sample is in fact heavily biased towards later types; over three-quarters are Hubble types Second or later. As an additional, secondary sample, we include 15 galaxies from Rossa et al. [11], where the masses are estimated by fits of multiple SSP models to high-resolution spectroscopy. Most of these galaxies are Sc and later, but a few earlier-type spirals $(\mathrm{Sa}-\mathrm{Sb})$ are also included.

Total stellar masses are based on $\mathrm{K}$-band total magnitudes from 2MASS [32] or from Malhotra et al. [33] for M31 and M33 (which are too large for accurate sky subtraction of 2MASS images), combined with color-based mass-to-light $(M / L)$ ratios from Bell et al. [34]. For the latter, we use optical colors from the literature (primarily from HyperLeda (http://leda.univ-lyon1.fr/)) or from direct measurements on Sloan Digital Sky Survey (SDSS, [35]) images. The bulge masses are derived using bulge-to-total $(B / T)$ values determined individually for each galaxy by $2 \mathrm{D}$ image decomposition, using the BUDDA software package $[36,37]$, which incorporates bulge and disk components and optional bars and central point sources (the latter can be used for both nuclear star clusters and AGN). Note that we explicitly define "bulge" to be the "photometric bulge", that is, the excess light (and stellar mass) which is not part of the disk, bar, or nuclear star cluster. We defer questions of how SMBH (or nuclear cluster) mass relates to so-called "pseudobulges" versus "classical bulges" (e.g., [38, 39]) to a later analysis.

Full 2D decompositions, as described above, were used for all S0 and spiral SMBH host galaxies. For the NSC host galaxies, we follow the same approach, with one simplification. Since we have found that $B / T$ ratios for unbarred galaxies do not change dramatically if we use 1D surfacebrightness profile decompositions instead of $2 \mathrm{D}$ image decompositions, we use the former for genuinely unbarred galaxies; we are careful to exclude (or separately model) the NSC contribution to the surface-brightness profile in these cases. Galaxies which do possess bars are subjected to full 2D decompositions; see the following section for details.

2.1. Bulge-Disk Decompositions. As noted previously, we use 2D image decompositions via the BUDDA software package to determine the $B / T$ ratios, and thus the bulge stellar masses, for SMBH host galaxies and for barred NSC host galaxies. For the NSC galaxies, we use HST data wherever possible, to enable the NSC itself to be properly modeled as a separate source. However, we have found that when the NSC is sufficiently luminous, and when the bulge is sufficiently low-contrast, we can achieve reasonable decompositions with ground-based images; these are sometimes preferable if they are near-IR (to minimize the effects of dust extinction and recent star formation) and/or large enough to include the entire galaxy (to allow better recovery of the disk component).

We have completed decompositions for the galaxies with dynamically determined NSC masses (we use the published 2D decomposition of [28] for NGC 3621); in the special case of the Milky Way, we assume a bulge mass of $\sim 1.0 \times$ $10^{10} M_{\odot}$ and a total stellar mass of $5.5 \times 10^{10} M_{\odot}$, based on arguments in Dehnen and Binney [40], Klypin et al. [41], and Flynn et al. [42]. Decompositions for the spectroscopic sample are still in progress but are mostly complete; since our primary analysis (e.g., computing the $M_{\mathrm{NSC}}-M_{\star \text {,bul }}$ relation) is based on the dynamical masses, the incompleteness of the spectroscopic sample does not affect our results.

Full details of the individual decompositions will be published elsewhere (Erwin and Gadotti 2012a, in prep.). An example of one of the 2D decompositions is given in Figure 1 for the galaxy NGC 7418, where we fit an $\mathrm{H}$-band image from the Ohio State University Bright Spiral Galaxy Survey [43, OSU BSGS] using an exponential disk $(95.6 \%$ of the light), a Sérsic bulge (1.6\% of the light), a bar ( $2.4 \%$ of the light), and a point source for the NSC ( $0.5 \%$ of the light). (In this particular galaxy, the disk appears to be truncated, but this has little effect on the decomposition; including a broken-exponential profile for the disk changes the $B / T$ ratio from 0.016 to 0.017 .) This illustrates the importance of including a separate bar component in the decomposition when the galaxy is barred; the bar has almost twice the luminosity of the bulge, and, without it, the bulge luminosity 


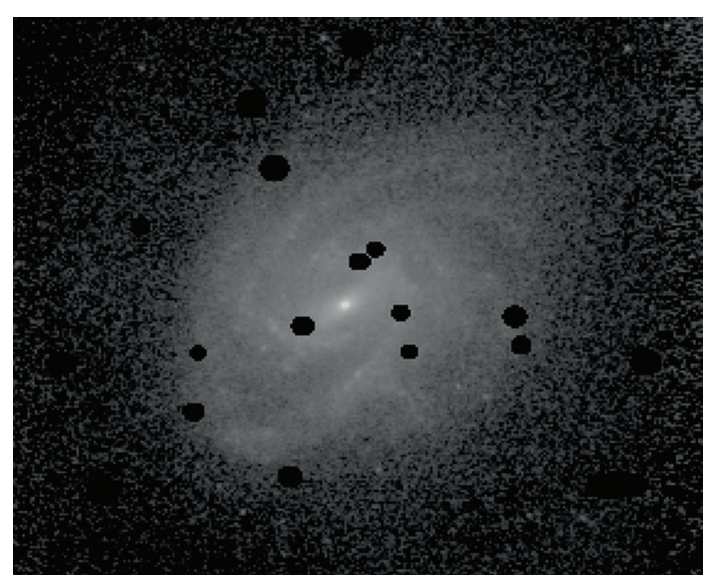

(a)

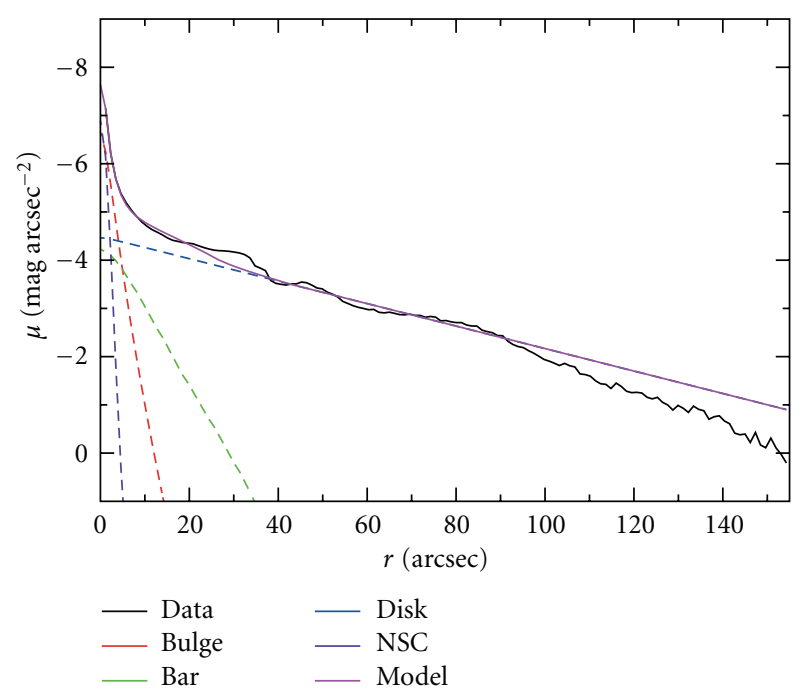

(c)

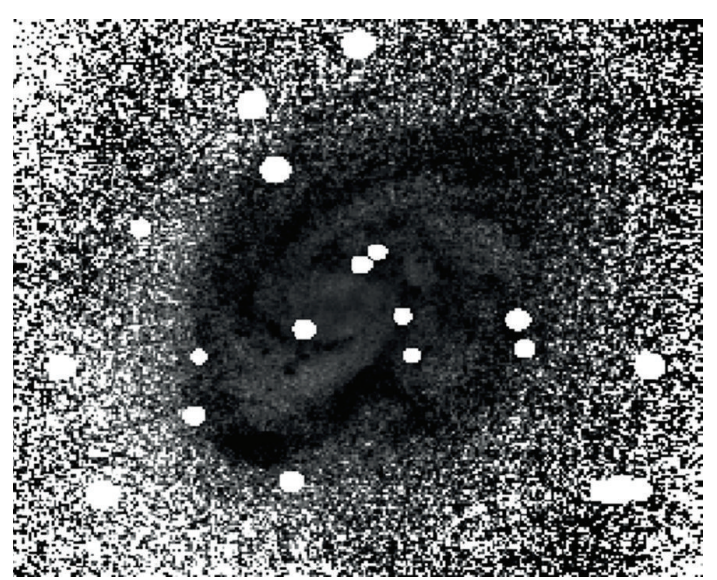

(b)

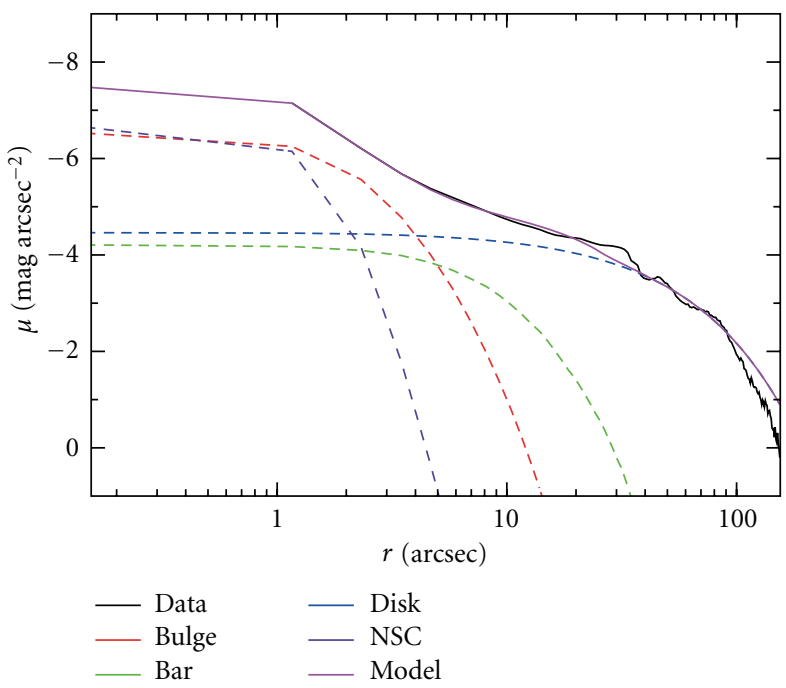

(d)

FIGURE 1: An example of one of our 2D decompositions of NSC host galaxies - in this instance, the decomposition of the OSU BSGS H-band image of NGC 7418, using an exponential disk, a Sérsic bulge $(n=1.5)$, a bar, and a point source for the NSC. (a): original $H$-band image, with masking of bright stars (logarithmic brightness scaling). (b): residual image after subtracting best-fitting model image. (c): major-axis profile (black) along with the components of the model and their sum (purple). (d): same, but plotted with logarithmic major-axis scaling.

(and stellar mass) would certainly be overestimated. In fact, a $1 \mathrm{D}$ decomposition for this galaxy gives a $B / T$ value almost twice as large (0.030); similar results were found for four other barred galaxies in the sample, with mean $B / T$ values a factor of 2.1 times larger when the bar was omitted; see also [37].

\section{Comparing Black Holes and Nuclear Star Clusters}

Although black-hole-bulge correlations are sometimes described as correlations between the black hole mass and the host galaxy mass (or luminosity as a proxy for mass), this is really only true for elliptical galaxies, where the entire galaxy is the "bulge." Kormendy and Gebhardt [17] explicitly compared $B$-band total and bulge luminosities for SMBH hosts and showed that the latter provided a much better correlation. Most recently, Kormendy et al. [18] have show for a larger, updated sample that $\mathrm{SMBH}$ masses in disk galaxies correlated much better with (classical) bulge $K$-band luminosity than with the luminosity of the disk component; this naturally suggests that total-galaxy luminosity is unlikely to correlate well with SMBH mass when the galaxy is disk dominated.

In Figure 2, we compare SMBH masses with total galaxy stellar mass (Figure 2(a)) and with bulge stellar mass (Figure 2(b)), based on our careful bulge/disk/bar decompositions (see Table 1). Error bars include the effects of uncertainties in the distance and in the $M / L$ and $B / T$ ratios. As expected, the correlation between SMBH mass and bulge mass is much stronger than any correlation with total galaxy mass; the Spearman correlation coefficients are $r_{S}=0.71$ for the $M_{\mathrm{BH}}-M_{\star \text {, bul }}$ relation versus 0.29 for the $M_{\mathrm{BH}}-M_{\star \text {,tot }}$ 


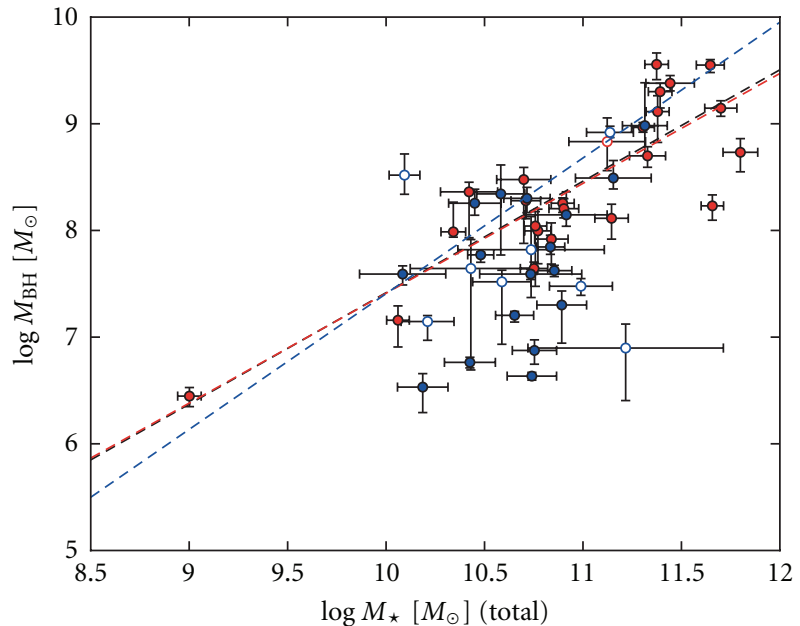

(a)

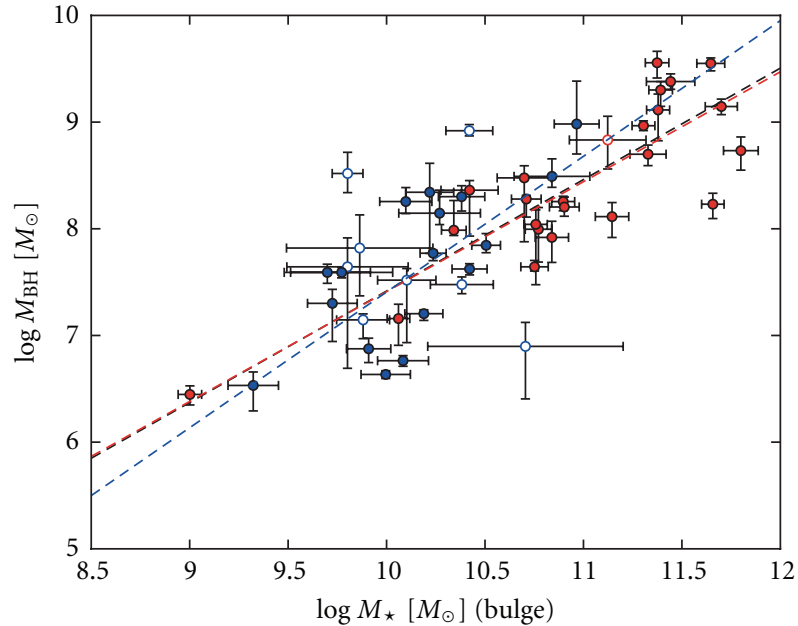

(b)

FIGURE 2: (a): SMBH mass (red: elliptical galaxies, blue: disk galaxies) versus total galaxy stellar mass. (b): SMBH mass versus bulge stellar mass. (Data and sources in Table 1). The diagonal dashed lines are the best fits to the $M_{\mathrm{BH}}-M_{\star, \text { bul }}$ relation for the whole sample (black), for the elliptical galaxies (red), and for bulges of the disk galaxies (blue). Open symbols are galaxies without precise distances, which are not used in the fits. It is clear that the SMBH masses of S0 and spiral galaxies (blue) correlate better with the bulge stellar mass than with total galaxy mass.

relation, with the latter correlation not being statistically significant.

We also plot linear fits of $\log M_{\mathrm{BH}}$ as a function of $\log M_{\star \text {,bul }}$; these fits are made using galaxies with welldetermined distances (filled points) to minimize distancebased uncertainties, using the Bayesian-based approach of D'Agostini [44], which explicitly incorporates errors in both variables and intrinsic scatter in the black hole mass (see also $[45,46])$. By "well-determined distances," we mean those determined using direct methods such as surface-brightness fluctuations and Cepheid stars, or redshift-based distances, where $z>0.01$ (to avoid large relative uncertainties due to peculiar motions.) The best-fitting relation for the whole sample (black line; the fit to just the elliptical galaxies, shown by the red line, is almost identical) is

$$
\begin{aligned}
\log M_{\mathrm{BH}}= & 8.46 \pm 0.08 \\
& +(1.04 \pm 0.12) \log \left(M_{\star, \mathrm{bul}} / 10^{11} M_{\odot}\right),
\end{aligned}
$$

with intrinsic scatter in SMBH mass of $0.39 \pm 0.05$ dex; the best-fitting relation for the bulges of disk galaxies only (blue line) is

$$
\begin{aligned}
\log M_{\mathrm{BH}}= & 8.68 \pm 0.20 \\
& +(1.27 \pm 0.26) \log \left(M_{\star, \text { bul }} / 10^{11} M_{\odot}\right),
\end{aligned}
$$

with intrinsic scatter $=0.41 \pm 0.07$. (The errors are based on bootstrap resampling.)

We apply exactly the same methodology to NSC-host galaxies in Figure 3, plotting NSC mass versus total galaxy stellar mass in the left panel and versus bulge stellar mass in the right panel. Since several of the NSC host galaxies are genuinely bulgeless systems (without even a distinct "pseudobulge"), we plot their bulge masses as upper limits $\left(B / T<0.001 M_{\star, \text { tot }}\right)$. As the figure shows, NSC mass clearly correlates better with total stellar mass than it does with bulge mass. (The respective correlation coefficients are $r_{S}=0.76$ versus 0.38 ; the bulge-mass correlation is not statistically significant.) Fitting NSC mass versus total stellar mass, using the same methodology as for the SMBH fits, gives the following relation:

$$
\begin{aligned}
\log M_{\mathrm{NSC}}= & 7.65 \pm 0.23 \\
& +(0.90 \pm 0.21) \log \left(M_{\star, \mathrm{tot}} / 10^{11} M_{\odot}\right),
\end{aligned}
$$

with intrinsic scatter $=0.43 \pm 0.10 \mathrm{dex}$. Note that the slope is formally indistinguishable from unity; that is, the $M_{\mathrm{NSC}} / M_{\star \text {,tot }}$ ratio does not appear to depend on $M_{\star \text {,tot }}$ itself.

It is important to note that the difference in correlation coefficients actually underestimates the true difference between the two relations, because the $M_{\mathrm{NSC}}-M_{\star \text {,bul }}$ correlation was computed assuming that bulgeless spirals still have nominal bulges (using $B / T=0.001$ ). In the combined sample of dynamical and spectroscopic NSC masses, we can identify at least three galaxies which have no detectable bulge. In two of these (NGC 1493 and NGC 2139), our 2D decomposition assigned stellar light to a bar in addition to a pure exponential disk; in 1D decompositions (or simple bulge + disk 2D decompositions), light from the bar might be (wrongly, we would argue) interpreted as "bulge" light. For the other galaxy (NGC 300), however, there is no ambiguity; this is an unbarred spiral galaxy with a surface brightness profile consisting of only an exponential disk and the NSC (see, e.g., Figure 8 of [47]).

The existence of nuclear star clusters in genuinely bulgeless spirals is simply an additional, direct confirmation 
TABLE 1: Galaxies with well-determined SMBH masses.

\begin{tabular}{|c|c|c|c|c|c|c|}
\hline Name & $T$ & $\begin{array}{c}D \\
(\mathrm{Mpc})\end{array}$ & $\begin{array}{l}M_{\mathrm{BH}}(+,-) \\
\left(\log _{10} M_{\odot}\right)\end{array}$ & Source & $\begin{array}{l}\text { Total } M_{\star}(\text { err }) \\
\quad\left(\log _{10} M_{\odot}\right)\end{array}$ & $\begin{array}{l}\text { Bulge } M_{\star}(\text { err }) \\
\quad\left(\log _{10} M_{\odot}\right)\end{array}$ \\
\hline Milky way & 4 & 0.01 & $6.63(+0.03,-0.04)$ & 1 & $10.74(0.09)$ & $10.00(0.13)$ \\
\hline M31 & 3 & 0.77 & $8.15(+0.22,-0.10)$ & 2 & $10.92(0.06)$ & $10.27(0.21)$ \\
\hline M32 & -5 & 0.79 & $6.45(+0.08,-0.10)$ & 3 & $9.00(0.06)$ & $9.00(0.06)$ \\
\hline NGC524 & -1 & 23.3 & $8.92(+0.04,-0.02)$ & 4 & $11.14(0.09)$ & $10.42(0.12)$ \\
\hline NGC821 & -5 & 23.4 & $7.92(+0.15,-0.23)$ & 5 & $10.84(0.08)$ & $10.84(0.08)$ \\
\hline NGC1023 & -1 & 11.1 & $7.62(+0.04,-0.04)$ & 6 & $10.85(0.08)$ & $10.42(0.09)$ \\
\hline NGC1068 & 3 & 14.3 & $6.90(+0.14,-0.21)$ & 7 & $11.22(0.49)$ & $10.71(0.50)$ \\
\hline NGC1300 & 4 & 18.9 & $7.82(+0.29,-0.29)$ & 8 & $10.74(0.37)$ & $9.86(0.37)$ \\
\hline NGC1316 & -5 & 21.3 & $8.23(+0.10,-0.13)$ & 9 & $11.66(0.06)$ & $11.66(0.06)$ \\
\hline NGC1399 & -5 & 21.1 & $9.11(+0.15,-0.29)$ & 10 & $11.38(0.06)$ & $11.38(0.06)$ \\
\hline NGC2549 & -2 & 12.3 & $7.15(+0.02,-0.16)$ & 4 & $10.21(0.12)$ & $9.88(0.13)$ \\
\hline NGC2748 & 4 & 23.1 & $7.64(+0.25,-0.74)$ & 8 & $10.43(0.30)$ & $9.80(0.31)$ \\
\hline NGC2787 & -1 & 7.28 & $7.59(+0.04,-0.06)$ & 11 & $10.08(0.15)$ & $9.70(0.22)$ \\
\hline NGC3031 & 1 & 3.63 & $7.85(+0.11,-0.07)$ & 12 & $10.83(0.06)$ & $10.51(0.07)$ \\
\hline NGC3227 & 1 & 22.9 & $7.30(+0.13,-0.35)$ & 13 & $10.89(0.11)$ & $9.72(0.13)$ \\
\hline NGC3245 & -1 & 20.3 & $8.30(+0.10,-0.12)$ & 14 & $10.72(0.09)$ & $10.38(0.12)$ \\
\hline NGC3368 & 2 & 10.5 & $6.88(+0.09,-0.12)$ & 15 & $10.75(0.09)$ & $9.91(0.11)$ \\
\hline NGC3377 & -5 & 10.9 & $7.99(+0.28,-0.05)$ & 5 & $10.34(0.06)$ & $10.34(0.06)$ \\
\hline NGC3379 & -5 & 10.3 & $8.00(+0.20,-0.31)$ & 5 & $10.77(0.07)$ & $10.77(0.07)$ \\
\hline NGC3384 & -1 & 11.3 & $7.20(+0.03,-0.05)$ & 5 & $10.65(0.08)$ & $10.19(0.10)$ \\
\hline NGC3393 & 1 & 48.3 & $7.48(+0.03,-0.03)$ & 16 & $10.99(0.15)$ & $10.38(0.16)$ \\
\hline NGC3489 & -1 & 11.7 & $6.76(+0.04,-0.04)$ & 15 & $10.43(0.08)$ & $10.08(0.13)$ \\
\hline NGC3585 & -3 & 19.5 & $8.49(+0.16,-0.09)$ & 17 & $11.15(0.09)$ & $10.84(0.19)$ \\
\hline NGC3607 & -5 & 22.2 & $8.11(+0.13,-0.19)$ & 17 & $11.15(0.08)$ & $11.15(0.08)$ \\
\hline NGC3608 & -5 & 22.3 & $8.28(+0.02,-0.16)$ & 5 & $10.71(0.08)$ & $10.71(0.08)$ \\
\hline NGC3998 & -2 & 13.7 & $8.34(+0.27,-0.56)$ & 18 & $10.58(0.09)$ & $10.22(0.12)$ \\
\hline NGC4026 & -3 & 13.2 & $8.26(+0.12,-0.09)$ & 17 & $10.45(0.12)$ & $10.10(0.13)$ \\
\hline NGC4151 & 2 & 14.5 & $7.52(+0.10,-0.56)$ & 19 & $10.59(0.13)$ & $10.10(0.15)$ \\
\hline NGC4258 & 4 & 7.18 & $7.59(+0.04,-0.04)$ & 20 & $10.73(0.08)$ & $9.77(0.26)$ \\
\hline NGC4261 & -5 & 30.8 & $8.70(+0.08,-0.10)$ & 21 & $11.33(0.09)$ & $11.33(0.09)$ \\
\hline NGC4291 & -5 & 25.5 & $8.48(+0.10,-0.57)$ & 5 & $10.70(0.14)$ & $10.70(0.14)$ \\
\hline NGC4342 & -1 & 16.7 & $8.52(+0.20,-0.18)$ & 22 & $10.09(0.07)$ & $9.80(0.08)$ \\
\hline NGC4374 & -5 & 18.5 & $8.97(+0.04,-0.04)$ & 23 & $11.30(0.06)$ & $11.30(0.06)$ \\
\hline NGC4473 & -5 & 15.3 & $8.04(+0.13,-0.56)$ & 5 & $10.76(0.06)$ & $10.76(0.06)$ \\
\hline NGC4486 & -5 & 16.7 & $9.56(+0.11,-0.14)$ & 24 & $11.37(0.06)$ & $11.37(0.06)$ \\
\hline NGC4486A & -5 & 18.4 & $7.16(+0.13,-0.25)$ & 25 & $10.06(0.06)$ & $10.06(0.06)$ \\
\hline NGC4564 & -3 & 15.9 & $7.77(+0.02,-0.07)$ & 5 & $10.48(0.06)$ & $10.24(0.07)$ \\
\hline NGC4649 & -5 & 16.4 & $9.30(+0.08,-0.15)$ & 26 & $11.39(0.06)$ & $11.39(0.06)$ \\
\hline NGC4697 & -5 & 12.5 & $8.26(+0.05,-0.09)$ & 5 & $10.90(0.06)$ & $10.90(0.06)$ \\
\hline NGC5077 & -5 & 37.5 & $8.83(+0.21,-0.23)$ & 27 & $11.12(0.19)$ & $11.12(0.19)$ \\
\hline NGC5128 & -5 & 3.42 & $7.64(+0.06,-0.03)$ & 28 & $10.75(0.07)$ & $10.75(0.07)$ \\
\hline NGC5252 & -2 & 92.9 & $8.98(+0.40,-0.27)$ & 29 & $11.31(0.09)$ & $10.97(0.11)$ \\
\hline NGC5576 & -5 & 24.8 & $8.20(+0.09,-0.08)$ & 17 & $10.90(0.08)$ & $10.90(0.08)$ \\
\hline NGC5845 & -5 & 25.2 & $8.36(+0.07,-0.41)$ & 5 & $10.42(0.15)$ & $10.42(0.15)$ \\
\hline NGC6251 & -5 & 95.9 & $8.73(+0.12,-0.18)$ & 30 & $11.80(0.09)$ & $11.80(0.09)$ \\
\hline NGC7052 & -5 & 67.9 & $8.58(+0.23,-0.22)$ & 31 & $11.49(0.12)$ & $11.49(0.12)$ \\
\hline NGC7457 & -1 & 12.9 & $6.53(+0.12,-0.23)$ & 5 & $10.19(0.10)$ & $9.32(0.13)$ \\
\hline IC1459 & -5 & 28.4 & $9.38(+0.05,-0.04)$ & 32 & $11.44(0.12)$ & $11.44(0.12)$ \\
\hline IC4296 & -5 & 53.2 & $9.15(+0.06,-0.07)$ & 33 & $11.70(0.08)$ & $11.70(0.08)$ \\
\hline A1836-BCG & -5 & 155.6 & $9.55(+0.05,-0.06)$ & 33 & $11.65(0.07)$ & $11.65(0.07)$ \\
\hline
\end{tabular}

(1) Galaxy name. (2) Hubble type T from RC3. (3) Adopted distance in Mpc. (4) Logarithm of SMBH mass and uncertainties; masses have been rescaled using the distances column 2, if necessary. Uncertainties are 1- $\sigma$ values. (5) Source of SMBH measurement. (6) Logarithm of total galaxy stellar mass and uncertainty (see text for details). (7) Logarithm of bulge stellar mass and uncertainty, based on 2D decompositions in Erwin and Gadotti (2012b, in prep). 

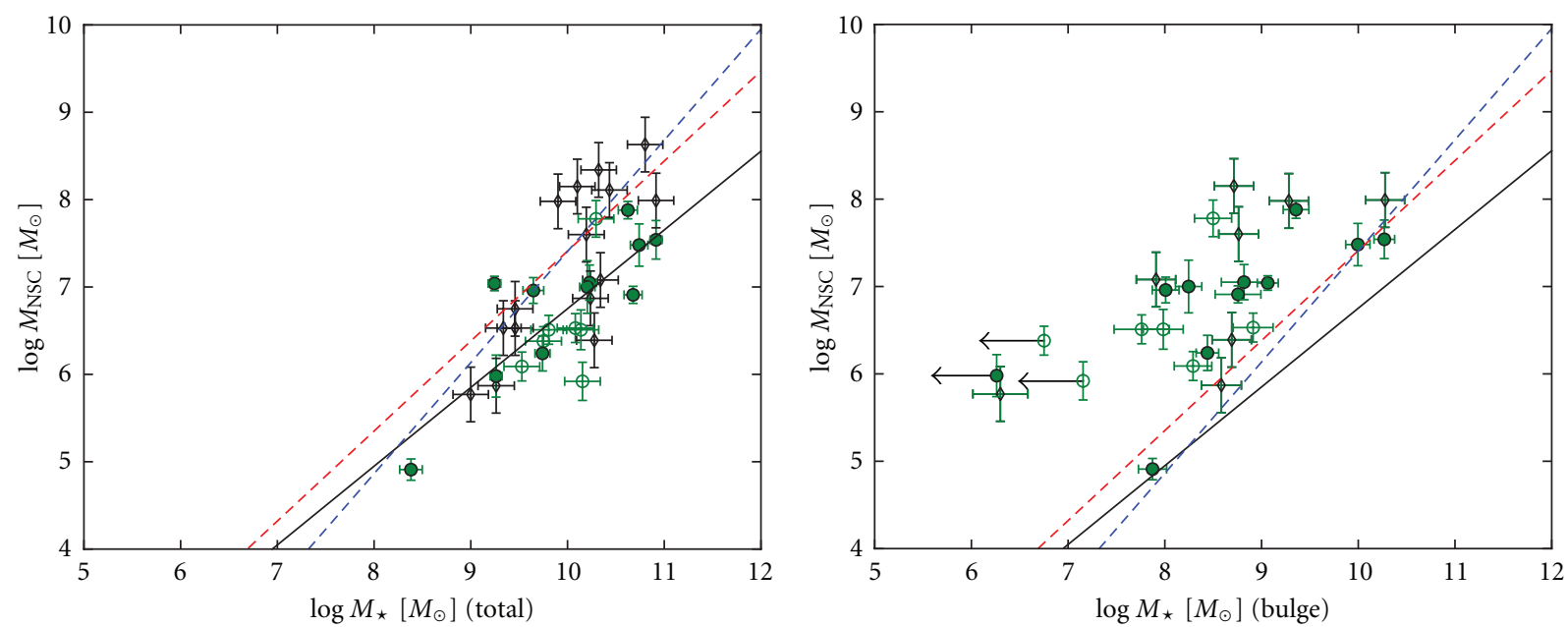

Figure 3: As for Figure 2, but now plotting NSC mass versus total stellar mass (a) and bulge stellar mass (b) (Data and sources in Table 2). Green circles are galaxies with dynamical mass estimates for their NSCs; black diamonds are the spectroscopically estimated masses of Rossa et al. [11] (bulge mass estimates are not complete for these galaxies). Filled symbols indicate galaxies with direct distance measurements (e.g., from Cepheid stars). Arrows show nominal upper limits for three bulgeless spirals (assuming that B/T $\leq 0.001$ ). The diagonal black line is a fit of NSC mass to total stellar mass for the dynamical-mass sample (green circles); for comparison, the diagonal dashed red and blue lines are the $M_{\mathrm{BH}}-M_{\star, \text { bul }}$ fits for ellipticals (red) and disk galaxies (blue) from Figure 2. The situation is now the reverse of that for SMBHs: NSC masses clearly correlate better with total galaxy mass than they do with bulge mass.

of our basic conclusion: nuclear star cluster masses scale with the total stellar mass of their host galaxies, not with the bulge mass. This means that NSCs and SMBHs do not follow a common host-galaxy correlation.

We have also investigated whether other galaxy parameters might correlate with NSC mass, or even with residuals from the $M_{\mathrm{NSC}}-M_{\star \text {, tot }}$ relation. In particular, we have compared NSC mass with rotation velocity and with total baryonic mass (stellar mass plus atomic gas from Hi measurements). In both cases, correlations exist, but they are not as strong as the correlation with total stellar mass. No particular correlations with residuals of the $M_{\mathrm{NSC}}-M_{\star \text {, tot }}$ relation are seen.

\section{Trends with Hubble Type}

Closer inspection of the left-hand panel of Figure 3 suggests that the spectroscopic masses (black diamonds) tend to be offset from the NSC- $M_{\star \text {, tot }}$ relation, in the sense that they have larger NSC masses for the same total stellar mass. This could, in principle, be evidence of a systematic overestimation of NSC masses in the spectroscopic sample, but of the four galaxies in common between Walcher et al. [21] and Rossa et al. [11] only one has a (slightly) higher spectroscopic mass, while the other three have spectroscopic masses slightly lower than the dynamical masses. There is, however, another difference to consider; the spectroscopic sample tends to have earlier Hubble types.

This brings us to something which Seth et al. [48] pointed out several years ago, using a larger dataset of NSCs and host galaxies, with NSC masses based (mostly) on colors or assumed $M / L$ ratios. They noted that NSCs in late-type spirals tended to have lower relative masses $\left(M_{\mathrm{NSC}} / M_{\star}\right.$, tot $)$ than early-type spirals and ellipticals. (Rossa et al. [11] pointed out a similar trend in absolute NSC mass for their smaller sample of NSCs in early- and late-type spirals.) Figure 4 makes this explicit by plotting $M_{\mathrm{NSC}} / M_{\star \text {, tot }}$ versus Hubble type for the galaxies in Seth et al.'s compilation, plus seven galaxies from our updated dynamical-mass sample which were not in their sample. We have also added galaxy stellar-mass estimates for 16 galaxies that did not have masses in Seth et al., using total $K$-band magnitudes from 2MASS and either $B-V$ colors from HyperLeda or measured $g-r$ colors from SDSS images to derive the $K$-band $M / L$ via Bell et al. [34].

What is curious about Figure 4 is not just that the $M_{\mathrm{NSC}} /$ $M_{\star \text {,tot }}$ ratio depends on Hubble type, but that it actually appears to do so in a bimodal fashion; Hubble types $\mathrm{Sb}$ and earlier have relatively large NSC masses, while Sc and latertype galaxies have significantly smaller relative NSC masses. Plotted on top of the figure are simple fits of a function where the $M_{\mathrm{NSC}} / M_{\star \text {, tot }}$ ratio can take two constant values, one for Hubble types $T<T_{1}$ and the other for $T>T_{2}$, with a simple linear transition between $T_{1}$ and $T_{2}$. Fits to just the dynamical + spectroscopic masses (red dashed line) and to the entire sample (gray dashed line) are similar, indicating that $\mathrm{Sb}$ and earlier Hubble types form one class, with $\left\langle M_{\mathrm{NSC}} / M_{\star, \text { tot }}\right\rangle \sim 0.002$, and Sc and later types form a different group, with $\left\langle M_{\mathrm{NSC}} / M_{\star, \text { tot }}\right\rangle$ almost an order of magnitude smaller $(\sim 0.0003)$. The corresponding best-fit values of $\left(T_{1}, T_{2}\right)$ are $(3.51,4.05)$ for the dynamical + spectroscopic masses and $(3.10,5.01)$ for the complete sample. As a crude check on whether this split is statistically significant, we performed Kolmogorov-Smirnov tests on the values of $M_{\mathrm{NSC}} / M_{\star \text {,tot }}$ for galaxies with $T \leq 3$ and galaxies with $T \geq 5$. The K-S test gives a probability $P_{\mathrm{KS}}=0.0038$ 
TABLE 2: Galaxies with well-determined NSC masses.

\begin{tabular}{|c|c|c|c|c|c|c|c|}
\hline Name & $T$ & $\begin{array}{c}D \\
(\mathrm{Mpc})\end{array}$ & $\begin{array}{l}M_{\mathrm{NSC}}(\mathrm{err}) \\
\left(\log _{10} M_{\odot}\right)\end{array}$ & Type & Source & $\begin{array}{c}\text { Total } M_{\star}(\text { err }) \\
\left(\log _{10} M_{\odot}\right)\end{array}$ & $\begin{array}{c}\text { Bulge } M_{\star}(\text { err }) \\
\left(\log _{10} M_{\odot}\right) \\
\end{array}$ \\
\hline Milky way & 4 & 0.01 & $7.48(0.09)$ & $\mathrm{D}$ & 1 & $10.74(0.09)$ & $10.00(0.13)$ \\
\hline M31 & 3 & 0.77 & $7.54(0.06)$ & $\mathrm{D}$ & 2 & $10.92(0.06)$ & $10.27(0.11)$ \\
\hline M33 & 6 & 0.81 & $6.24(0.08)$ & $\mathrm{D}$ & 3 & $9.74(0.08)$ & $8.44(0.12)$ \\
\hline IC342 & 6 & 3.37 & $7.05(0.07)$ & $\mathrm{D}$ & 4 & $10.23(0.07)$ & $8.82(0.23)$ \\
\hline NGC300 & 7 & 2.02 & $5.98(0.06)$ & $\mathrm{D}$ & 5 & $9.26(0.06)$ & $<6.26$ \\
\hline NGC404 & -3 & 3.18 & $7.04(0.06)$ & $\mathrm{D}$ & 6 & $9.24(0.06)$ & $9.06(0.11)$ \\
\hline NGC428 & 9 & 15.5 & $6.51(0.18)$ & $\mathrm{D}$ & 5 & $9.81(0.18)$ & $7.76(0.29)$ \\
\hline NGC1042 & 6 & 17.5 & $6.51(0.18)$ & $\mathrm{D}$ & 5 & $10.14(0.18)$ & $7.98(0.21)$ \\
\hline NGC1493 & 6 & 11.0 & $6.38(0.19)$ & $\mathrm{D}$ & 5 & $9.75(0.19)$ & $<6.75$ \\
\hline NGC1705 & -3 & 5.11 & $4.91(0.12)$ & $\mathrm{D}$ & 7 & $8.38(0.12)$ & $7.87(0.15)$ \\
\hline NGC2139 & 6 & 22.9 & $5.92(0.18)$ & $\mathrm{D}$ & 5 & $10.15(0.18)$ & $<7.15$ \\
\hline NGC3423 & 6 & 14.4 & $6.53(0.19)$ & $\mathrm{D}$ & 5 & $10.08(0.19)$ & $8.91(0.21)$ \\
\hline NGC3621 & 7 & 6.64 & $7.00(0.08)$ & $\mathrm{D}$ & 8 & $10.20(0.08)$ & $8.25(0.14)$ \\
\hline NGC5457 & 6 & 7.05 & $6.91(0.09)$ & $\mathrm{D}$ & 9 & $10.68(0.09)$ & $8.76(0.24)$ \\
\hline NGC6946 & 6 & 5.89 & $7.88(0.10)$ & $\mathrm{D}$ & 9 & $10.62(0.10)$ & $9.36(0.13)$ \\
\hline NGC7418 & 6 & 17.8 & $7.78(0.18)$ & $\mathrm{D}$ & 5 & $10.29(0.18)$ & $8.50(0.19)$ \\
\hline NGC7424 & 6 & 10.5 & $6.09(0.18)$ & $\mathrm{D}$ & 5 & $9.53(0.18)$ & $8.29(0.19)$ \\
\hline NGC7793 & 7 & 3.91 & $6.96(0.11)$ & $\mathrm{D}$ & 5 & $9.65(0.11)$ & $8.01(0.14)$ \\
\hline NGC1325 & 4 & 19.6 & $7.08(0.18)$ & S & 10 & $10.34(0.18)$ & $7.91(0.20)$ \\
\hline NGC1385 & 6 & 18.1 & $6.39(0.18)$ & S & 10 & $10.28(0.18)$ & $8.69(0.20)$ \\
\hline NGC2552 & 9 & 9.68 & $5.77(0.18)$ & S & 10 & $9.00(0.18)$ & $6.30(0.28)$ \\
\hline NGC3177 & 3 & 19.6 & $8.15(0.18)$ & S & 10 & $10.10(0.18)$ & $8.71(0.20)$ \\
\hline NGC3277 & 2 & 21.4 & $8.34(0.18)$ & S & 10 & $10.32(0.18)$ & $\ldots$ \\
\hline NGC3455 & 3 & 16.4 & $6.75(0.18)$ & S & 10 & $9.46(0.18)$ & $\ldots$ \\
\hline NGC4030 & 4 & 20.5 & $7.99(0.18)$ & $S$ & 10 & $10.92(0.18)$ & $10.28(0.20)$ \\
\hline NGC4411B & 6 & 18.6 & $6.53(0.19)$ & S & 10 & $9.46(0.19)$ & $\ldots$ \\
\hline NGC4701 & 6 & 10.8 & $6.53(0.18)$ & S & 10 & $9.34(0.18)$ & $\ldots$ \\
\hline NGC4775 & 7 & 21.9 & $7.60(0.19)$ & S & 10 & $10.19(0.19)$ & $8.76(0.20)$ \\
\hline NGC5377 & 1 & 28.4 & $8.63(0.18)$ & S & 10 & $10.80(0.18)$ & $\ldots$ \\
\hline NGC5585 & 7 & 8.71 & $5.87(0.19)$ & S & 10 & $9.26(0.19)$ & $8.58(0.21)$ \\
\hline NGC5806 & 3 & 20.0 & $8.11(0.18)$ & S & 10 & $10.43(0.18)$ & $\ldots$ \\
\hline NGC7421 & 4 & 23.1 & $6.87(0.18)$ & $S$ & 10 & $10.24(0.18)$ & $\ldots$ \\
\hline NGC7690 & 3 & 17.7 & $7.98(0.18)$ & $S$ & 10 & $9.90(0.18)$ & $9.28(0.20)$ \\
\hline
\end{tabular}

(1) Galaxy name. (2) Hubble type T from RC3. (3) Adopted distance in Mpc. (4) Logarithm of NSC mass and uncertainty; masses have been rescaled using the distances column 2, if necessary. Errors are 1- $\sigma$ values. (5) Type of NSC mass measurement: D: dynamical, S: spectroscopic. (6) Source of NSC measurement: 1 = Launhardt et al. [31]; $2=$ Kormendy and Bender [25]; $3=$ Matthews et al. [26] + Gebhardt et al. [27]; $4=$ Böker et al. [24]; $5=$ Walcher et al. [21]; $6=$ Seth et al. [29]; 7 = Ho and Filippenko [23]; $8=$ Barth et al. [28]; $9=$ Kormendy et al. [30]; $10=$ Rossa et al. [11]. (7) Logarithm of total galaxy stellar mass and uncertainty (see text for details). (8) Logarithm of bulge stellar mass and uncertainty (or upper limit for bulgeless galaxies), based on decompositions in Erwin and Gadotti (2012a, in prep); galaxies currently missing proper decompositions are indicated by “...”.

for the two sets of ratios coming from the same parent population if we use only the dynamical + spectroscopic masses, or $P_{\mathrm{KS}}=3.1 \times 10^{-10}$ if we use the entire set of NSC masses.

Do NSCs in late-type spirals differ from those in earlytype spirals, S0s, and ellipticals in any sense other than average mass? The available evidence is ambiguous. Böker [7] notes that NSC sizes appear to be independent of Hubble type. On the other hand, Rossa et al. [11] compared stellar populations of NSCs in early- and late-type spirals using fits to their spectroscopy and noted that the NSCs in latetype spirals did tend to have younger stellar populations and (slightly) lower metallicities. (They also argued against any observational effects that might produce systematic overestimates of NSC mass in early spirals.) This does at least suggest that different star-formation histories may lie behind the mass differences in NSCs.

We also plot the $M_{\mathrm{BH}} / M_{\star}$,bul ratio for SMBH host galaxies (thick gray dotted line in Figure 4, based on (1)). What this indicates is that the NSC-host-galaxy relationship for $\mathrm{Sb}$ and earlier types is consistent with the $\mathrm{SMBH}$ relation, if all of the galaxy mass is in the bulge. Since most of the galaxies used for the original CMO studies $[8,9]$ were dwarf and giant ellipticals (or $S 0$ galaxies with high $B / T$ ratios), it is easy to 


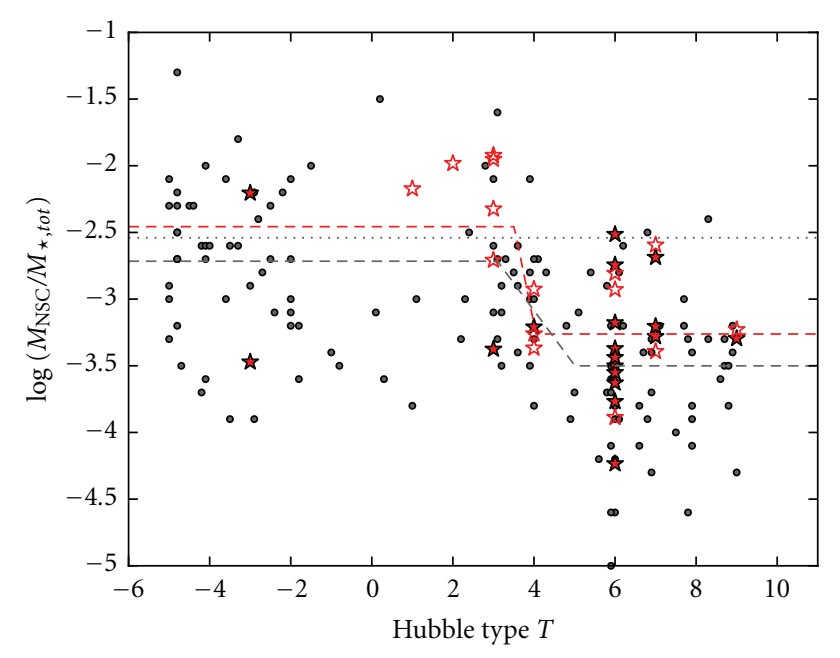

FIgUre 4: Relative masses of NSCs versus Hubble type of host galaxy, based on the compilation of Seth et al. [48]. Filled gray circles are NSC masses estimated from broadband colors or assumed $M / L$ ratios by Seth et al [29]; red stars indicate NSC masses from spectroscopic (hollow) or dynamical (filled) measurements (see Table 2 for references). Also shown are simple fits to the dynamical+spectroscopic masses (dashed red line) and to the entire sample (dashed gray line), along with the mean mass ratio of SMBHs relative to their host bulges (dotted gray line).

see why the "NSC = SMBH" connection could be made. But this is clearly true only for very bulge-dominated systems.

\section{Appendix}

\section{Data Tables}

In Tables 1 and 2, we list the basic data parameters for SMBH and NSC hosts. References for the NSC masses are in the captions for Table 2. For the SMBH masses, the numbers in column 5 of Table 1 translate into the following references: $1=$ Gillessen et al. [49]; 2 = Bender et al. [50]; 3 = Verolme et al. [51]; $4=$ Krajnović et al. [52]; $5=$ Gebhardt et al. [53]; $6=$ Bower et al. [54]; 7 = Lodato and Bertin [55]; $8=$ Atkinson et al. [56]; $9=$ Rusli et al. [57]; $10=$ Nowak et al. [58]; $11=$ Houghton et al. [59]; $12=$ Sarzi et al. [60]; $13=$ Devereux et al. [61]; $14=$ Davies et al. [62]; $15=$ Barth et al. [63]; $16=$ Nowak et al. [39]; 17 = Kondratko et al. [64]; $18=$ Güeltekin et al. [65]; $19=$ de Francesco et al. [66]; $20=$ Hicks and Malkan [67]; 21 = Miyoshi et al. [68]; $22=$ Ferrarese et al. [69]; 23 = Cretton and van den Bosch [70]; $24=$ Walsh et al. [71]; $25=$ Macchetto et al. [72]; $26=$ Nowak et al. [73]; $27=$ Shen and Gebhardt [74]; $28=$ de Francesco et al. [75]; $29=$ Neumayer et al. [76]; $30=$ Capetti et al. [77]; $31=$ Ferrarese and Ford [78]; 32 = van der Marel and van der Bosch [79]; 33 = Cappellari et al. [80]; $34=$ Bontà et al [81].

\section{Acknowledgments}

The authors would like to thank Eva Noyola and Anil Seth for useful and interesting conversations, along with comments from two referees which improved the manuscript. They would also like to thank the organizers of the 2008 "Nuclear Star Clusters across the Hubble Sequence" workshop in Heidelberg and the 2010 "ESO Workshop on Central Massive Objects" in Garching for helping motivate and inspire this research. This work was supported by Priority Programme 1177 ("Witnesses of Cosmic History: Formation and evolution of black holes, galaxies and their environment") of the Deutsche Forschungsgemeinschaft. This work made use of data from the Ohio State University Bright Spiral Galaxy Survey, which was funded by Grants AST-9217716 and AST9617006 from the United States National Science Foundation, with additional support from Ohio State University. It is also based on observations made with the NASA/ESA Hubble Space Telescope, obtained from the data archive at the Space Telescope Science Institute. STScI is operated by the Association of Universities for Research in Astronomy, Inc. under NASA contract NAS 5-26555. Funding for the creation and distribution of the SDSS Archive has been provided by the Alfred P. Sloan Foundation, the Participating Institutions, the National Aeronautics and Space Administration, the National Science Foundation, the U.S. Department of Energy, the Japanese Monbukagakusho, and the Max Planck Society. The SDSS Web site is http://www.sdss.org/. The SDSS is managed by the Astrophysical Research Consortium (ARC) for the Participating Institutions. The Participating Institutions are The University of Chicago, Fermilab, the Institute for Advanced Study, the Japan Participation Group, The Johns Hopkins University, the Korean Scientist Group, Los Alamos National Laboratory, the Max-Planck-Institute for Astronomy (MPIA), the Max-Planck-Institute for Astrophysics (MPA), New Mexico State University, University of Pittsburgh, University of Portsmouth, Princeton University, the United States Naval Observatory, and the University of Washington. Finally, this research made use of the LyonMeudon Extragalactic Database (LEDA; part of HyperLeda at http://leda.univ-lyon1.fr/), and the NASA/IPAC Extragalactic Database (NED), which is operated by the Jet Propulsion Laboratory, California Institute of Technology, under contract with the National Aeronautics and Space Administration.

\section{References}

[1] L. Ferrarese and D. Merritt, "A fundamental relation between supermassive black holes and their host galaxies," The Astrophysical Journal, vol. 539, no. 1, pp. L9-L12, 2000.

[2] K. Gebhardt, R. Bender, G. Bower et al., "A relationship between nuclear black hole mass and galaxy velocity dispersion," The Astrophysical Journal, vol. 539, no. 1, pp. L13-L16, 2000.

[3] A. Marconi and L. K. Hunt, "The relation between black hole mass, bulge mass, and near-Infrared luminosity," The Astrophysical Journal, vol. 589, no. 1, pp. L21-L24, 2003.

[4] N. Häring and H.-W. Rix, "On the black hole mass-bulge mass relation," The Astrophysical Journal, vol. 604, pp. L89L92, 2004.

[5] C. M. Carollo, M. Stiavelli, P. T. de Zeeuw, and J. Mack, "Spiral galaxies with WFPC2. I. Nuclear morphology, bulges, 
star clusters, and surface brightness profiles," The Astronomical Journal, vol. 114, no. 6, pp. 2366-2380, 1997.

[6] T. Böker, S. Laine, R. P. van der Marel et al., "A hubble space telescope census of nuclear star clusters in late-type spiral galaxies. I. Observations and image analysis," The Astronomical Journal, vol. 123, no. 3, pp. 1389-1410, 2002.

[7] T. Böker, "Properties of nuclear star clusters," Journal of Physics Conference Series, vol. 131, no. 1, Article ID 012043, 2008.

[8] E. H. Wehner and W. E. Harris, "From supermassive black holes to dwarf elliptical nuclei: a mass continuum," The Astrophysics Journal, vol. 644, pp. L17-L20, 2006.

[9] L. Ferrarese, P. Côté, E. Dalla Bontà et al., "A fundamental relation between compact stellar nuclei, supermassive black holes, and their host galaxies," The Astrophysical Journal Letters, vol. 644, no. 1, pp. L21-L24, 2006.

[10] P. Côté, S. Piatek, L. Ferrarese et al., "The ACS virgo cluster survey. VIII. The nuclei of early-type galaxies," The Astrophysical Journal Supplement Series, vol. 165, no. 1, pp. 57-94, 2006.

[11] J. Rossa, R. P. van der Marel, T. Böker et al., "Hubble space telescope stis spectra of nuclear star clusters in spiral galaxies: dependence of age and mass on hubble type," The Astronomical Journal, vol. 132, no. 3, pp. 1074-1099, 2006.

[12] M. Balcells, A. W. Graham, and R. F. Peletier, "Galactic bulges from hubble space telescope NICMOS observations: central galaxian objects, and nuclear profile slopes," The Astrophysical Journal, vol. 665, no. 2, pp. 1084-1103, 2007.

[13] D. E. McLaughlin, A. R. King, and S. Nayakshin, "The M- $\sigma$ relation for nucleated galaxies," The Astrophysical Journal, vol. 650, no. 1, pp. L37-L40, 2006.

[14] Y. Li, Z. Haiman, and M.-M. Mac Low, "Correlations between central massive objects and their host galaxies: from bulgeless spirals to ellipticals," The Astrophysical Journal, vol. 663, no. 1 I, pp. 61-70, 2007.

[15] S. Nayakshin, M. I. Wilkinson, and A. King, "Competitive feedback in galaxy formation," Monthly Notices of the Royal Astronomical Society, vol. 398, pp. L54-L57, 2009.

[16] B. Devecchi, M. Volonteri, M. Colpi, and F. Haardt, "Highredshift formation and evolution of central massive objects - I. Model description," Monthly Notices of the Royal Astronomical Society, vol. 409, no. 3, pp. 1057-1067, 2010.

[17] J. Kormendy and K. Gebhardt, "Supermassive black holes in galactic nuclei," in 20th Texas Symposium on Relativistic Astrophysics, J. C. Wheeler and H. Martel, Eds., vol. 586 of American Institute of Physics Conference Series, pp. 363-381, 2001.

[18] J. Kormendy, R. Bender, and M. E. Cornell, "Supermassive black holes do not correlate with galaxy disks or pseudobulges," Nature, vol. 469, pp. 374-376, 2011.

[19] C. M. Carollo, M. Stiavelli, and J. Mack, "Spiral galaxies with WFPC2. II. The nuclear properties of 40 objects," The Astronomical Journal, vol. 116, no. 1, pp. 68-84, 1998.

[20] K. Gültekin, D. O. Richstone, K. Gebhardt et al., "The M- $\sigma$ and $\mathrm{M}-\mathrm{L}$ relations in galactic bulges, and determinations of their intrinsic scatter," The Astrophysical Journal, vol. 698, no. 1, pp. 198-221, 2009.

[21] C. J. Walcher, R. P. van der Marel, D. Mclaughlin et al., "Masses of star clusters in the nuclei of bulgeless spiral galaxies," The Astrophysical Journal, vol. 618, no. 1, pp. 237-246, 2005.

[22] A. C. Seth, J. J. Dalcanton, P. W. Hodge, and V. P. Debattista, "Clues to nuclear star cluster formation from edge-on spirals," The Astronomical Journal, vol. 132, no. 6, pp. 2539-2555, 2006.

[23] L. C. Ho and A. V. Filippenko, "High-dispersion spectroscopy of a luminous, young star cluster in NGC 1705: further evidence for present-day formation of globular clusters," The Astrophysical Journal, vol. 472, no. 2, pp. 600-610, 1996.

[24] T. Böker, R. P. van der Marel, and W. D. Vacca, "CO band head spectroscopy of IC 342: mass and age of the nuclear star cluster 1," The Astronomical Journal, vol. 118, no. 2, pp. 831$842,1999$.

[25] J. Kormendy and R. Bender, "The double nucleus and central black hole of M31," The Astrophysical Journal, vol. 522, no. 2, pp. 772-792, 1999.

[26] L. D. Matthews, J. S. Gallagher III, J. E. Krist et al., "WFPC2 observations of compact star cluster nuclei in low-luminosity spiral galaxies," The Astronomical Journal, vol. 118, no. 1, pp. 208-235, 1999.

[27] K. Gebhardt, T. R. Lauer, J. Kormendy et al., "M33: a galaxy with no supermassive black hole," The Astronomical Journal, vol. 122, no. 5, pp. 2469-2476, 2001.

[28] A. J. Barth, L. E. Strigari, M. C. Bentz, J. E. Greene, and L. C. Ho, "Dynamical constraints on the masses of the nuclear star cluster and black hole in the late-type spiral galaxy NGC 3621," The Astrophysical Journal, vol. 690, no. 1, pp. 1031-1044, 2009.

[29] A. C. Seth, M. Cappellari, N. Neumayer et al., "The NGC 404 nucleus: star cluster and possible intermediate-mass black hole," The Astrophysical Journal, vol. 714, no. 1, pp. 713-731, 2010.

[30] J. Kormendy, N. Drory, R. Bender, and M. E. Cornell, "Bulgeless giant galaxies challenge our picture of galaxy formation by hierarchical clustering," The Astrophysical Journal, vol. 723, no. 1, pp. 54-80, 2010.

[31] R. Launhardt, R. Zylka, and P. G. Mezger, "The nuclear bulge of the galaxy III. Large-scale physical characteristics of stars and interstellar matter," Astronomy and Astrophysics, vol. 384, no. 1, pp. 112-139, 2002.

[32] T. H. Jarrett, T. Chester, R. Cutri, S. Schneider, M. Skrutskie, and J. P. Huchra, "2MASS extended source catalog: overview and algorithms," The Astronomical Journal, vol. 119, no. 5, pp. 2498-2531, 2000.

[33] S. Malhotra, D. N. Spergel, J. E. Rhoads, and L. I. Jing, "The milky way, local galaxies, and the infrared tully-fisher relation," The Astrophysical Journal, vol. 473, no. 2, pp. 687-691, 1996.

[34] E. F. Bell, D. H. McIntosh, N. Katz, and M. D. Weinberg, "The optical and near-infrared properties of galaxies. I. Luminosity and stellar MASS functions," The Astrophysical Journal Letters, vol. 149, no. 2, pp. 289-312, 2003.

[35] D. G. York, J. Adelman, J. E. Anderson Jr. et al., "The sloan digital sky survey: technical summary," The Astronomical Journal, vol. 120, no. 3, pp. 1579-1587, 2000.

[36] R. E. de Souza, D. A. Gadotti, and S. dos Anjos, "BUDDA: a new two-dimensional Bulge/Disk decomposition code for detailed structural analysis of galaxies," The Astrophysical Journal Supplement Series, vol. 153, no. 2, pp. 411-427, 2004.

[37] D. A. Gadotti, "Image decomposition of barred galaxies and AGN hosts," Monthly Notices of the Royal Astronomical Society, vol. 384, no. 1, pp. 420-439, 2008.

[38] J. Hu, "The black hole mass-stellar velocity dispersion correlation: bulges versus pseudo-bulges," Monthly Notices of the Royal Astronomical Society, vol. 386, no. 4, pp. 2242-2252, 2008.

[39] N. Nowak, J. Thomas, P. Erwin, R. P. Saglia, R. Bender, and R. I. Davies, "Do black hole masses scale with classical bulge luminosities only? The case of the two composite pseudobulge galaxies NGC 3368 and NGC 3489," Monthly Notices of the Royal Astronomical Society, vol. 403, no. 2, pp. 646-672, 2010. 
[40] W. Dehnen and J. Binney, "Mass models of the Milky Way," Monthly Notices of the Royal Astronomical Society, vol. 294, no. 3, pp. 429-438, 1998.

[41] A. Klypin, H. Zhao, and R. S. Somerville, " $\Lambda$ CDM-based models for the Milky Way and M31. I. Dynamical models," The Astrophysical Journal, vol. 573, no. 2, pp. 597-613, 2002.

[42] C. Flynn, J. Holmberg, L. Portinari, B. Fuchs, and H. Jahreiß, "On the mass-to-light ratio of the local Galactic disc and the optical luminosity of the Galaxy," Monthly Notices of the Royal Astronomical Society, vol. 372, no. 3, pp. 1149-1160, 2006.

[43] P. B. Eskridge, J. A. Frogel, R. W. Pogge et al., "Near-infrared and optical morphology of spiral galaxies," The Astrophysical Journal Supplement Series, vol. 143, no. 1, pp. 73-111, 2002.

[44] G. D'Agostini, "Fits, and especially linear fits, with errors on both axes, extra variance of the data points and other complications," Physics, 2005.

[45] C. Guidorzi, F. Frontera, E. Montanari et al., "The slope of the gamma-ray burst variability/peak luminosity correlation," Monthly Notices of the Royal Astronomical Society, vol. 371, pp. 843-851, 2006.

[46] O. Y. Gnedin, D. H. Weinberg, J. Pizagno, F. Prada, and H.-W. Rix, "Dark matter halos of disk galaxies: constraints from the Tully-Fisher relation," The Astrophysical Journal, vol. 671, no. 2, pp. 1115-1134, 2007.

[47] J. Bland-Hawthorn, M. Vlajić, K. C. Freeman, and B. T. Draine, "NGC 300: an extremely faint, outer stellar disk observed to 10 scale lengths," The Astrophysical Journal, vol. 629, no. 1, pp. 239-249, 2005.

[48] A. Seth, M. Agüeros, D. Lee, and A. Basu-Zych, "The coincidence of nuclear star clusters and active galactic nuclei," The Astrophysical Journal, vol. 678, no. 1, pp. 116-130, 2008.

[49] S. Gillessen, F. Eisenhauer, S. Trippe et al., "Monitoring stellar orbits around the massive black hole in the galactic center," The Astrophysical Journal, vol. 692, pp. 1075-1109, 2009.

[50] R. Bender, J. Kormendy, G. Bower et al., "HST STIS spectroscopy of the triple nucleus of M31: two nested disks in keplerian rotation around a supermassive black hole," The Astrophysical Journal, vol. 631, no. 1, pp. 280-300, 2005.

[51] E. K. Verolme, M. Cappellari, Y. Copin et al., "A SAURON study of M32: measuring the intrinsic flattening and the central black hole mass," Monthly Notices of the Royal Astronomical Society, vol. 335, pp. 517-525, 2002.

[52] D. Krajnović, R. M. McDermid, M. Cappellari, and R. L. Davies, "Determination of masses of the central black holes in NGC 524 and 2549 using laser guide star adaptive optics," Monthly Notices of the Royal Astronomical Society, vol. 399, no. 4, pp. 1839-1857, 2009.

[53] K. Gebhardt, D. Richstone, S. Tremaine et al., "Axisymmetric dynamical models of the central regions of galaxies," The Astrophysical Journal, vol. 583, no. 1, pp. 92-115, 2003.

[54] G. A. Bower, R. F. Green, R. Bender et al., "Evidence of a supermassive black hole in the galaxy NGC 1023 from the nuclear stellar dynamics," The Astrophysical Journal, vol. 550, no. 1, pp. 75-86, 2001.

[55] G. Lodato and G. Bertin, "Non-Keplerian rotation in the nucleus of NGC 1068: evidence for a massive accretion disk?" Astronomy and Astrophysics, vol. 398, no. 2, pp. 517-524, 2003.

[56] J. W. Atkinson, J. L. Collett, A. Marconi et al., "Supermassive black hole mass measurements for NGC 1300 and 2748 based on hubble space telescope emission-line gas kinematics," Monthly Notices of the Royal Astronomical Society, vol. 359, pp. 504-520, 2005.

[57] S. P. Rusli, J. Thomas, P. Erwin, R. P. Saglia, N. Nowak, and R. Bender, "The central black hole mass of the high- $\sigma$ but low-bulge-luminosity lenticular galaxy NGC 1332," Monthly Notices of the Royal Astronomical Society, vol. 410, no. 2, pp. 1223-1236, 2011.

[58] N. Nowak, R. P. Saglia, J. Thomas, R. Bender, R. I. Davies, and K. Gebhardt, "The supermassive black hole of FornaxA," Monthly Notices of the Royal Astronomical Society, vol. 391, pp. 1629-1649, 2008.

[59] R. C. W. Houghton, J. Magorrian, M. Sarzi, N. Thatte, R. L. Davies, and D. Krajnović, "The central kinematics of NGC 1399 measured with 14 pc resolution," Monthly Notices of the Royal Astronomical Society, vol. 367, no. 1, pp. 2-18, 2006.

[60] M. Sarzi, H.-W. Rix, J. C. Shields et al., "Supermassive black holes in bulges," The Astrophysical Journal, vol. 550, no. 1, pp. 65-74, 2001.

[61] N. Devereux, H. Ford, Z. Tsvetanov, and G. Jacoby, "STIS spectroscopy of the central 10 parsecs of M81: evidence for a massive black hole," The Astronomical Journal, vol. 125, no. 3, pp. 1226-1235, 2003.

[62] R. I. Davies, J. Thomas, R. Genzel et al., "The star-forming torus and stellar dynamical black hole mass in the Seyfert 1 nucleus of NGC 3227," The Astrophysical Journal, vol. 646, no. 2, pp. 754-773, 2006.

[63] A. J. Barth, M. Sarzi, H.-W. Rix, L. C. Ho, A. V. Filippenko, and W. L. W. Sargent, "Evidence for a supermassive black hole in the S0 galaxy NGC 3245," The Astrophysical Journal, vol. 555, no. 2, pp. 685-708, 2001.

[64] P. T. Kondratko, L. J. Greenhill, and J. M. Moran, "The parsecscale accretion disk in NGC 3393," The Astrophysical Journal, vol. 678, no. 1, pp. 87-95, 2008.

[65] K. Gültekin, D. O. Richstone, K. Gebhardt et al., "A quintet of black hole mass determinations," The Astrophysical Journal, vol. 695, pp. 1577-1590, 2009.

[66] G. de Francesco, A. Capetti, and A. Marconi, "Measuring supermassive black holes with gas kinematics: the active so galaxy NGC 3998," Astronomy and Astrophysics, vol. 460, no. 2, pp. 439-448, 2006.

[67] E. K. S. Hicks and M. A. Malkan, "Circumnuclear gas in seyfert 1 galaxies: morphology, kinematics, and direct measurement of black hole masses," The Astrophysical Journal Supplement Series, vol. 174, no. 1, pp. 31-73, 2008.

[68] M. Miyoshi, J. Moran, J. Herrnstein et al., "Evidence for a black hole from high rotation velocities in a sub-parsec region of NGC4258," Nature, vol. 373, no. 6510, pp. 127-129, 1995.

[69] L. Ferrarese, H. C. Ford, and W. Jaffe, "Evidence for a massive black hole in the active galaxy NGC 4261 from bubble space telescope images and spectra," The Astrophysical Journal, vol. 470, no. 1, pp. 444-459, 1996.

[70] N. Cretton and F. C. van den Bosch, "Evidence for a massive black hole in the S0 galaxy NGC 4342," The Astrophysical Journal, vol. 514, no. 2, pp. 704-724, 1999.

[71] J. L. Walsh, A. J. Barth, and M. Sarzi, "The supermassive black hole in M84 revisited," The Astrophysical Journal, vol. 721, no. 1, pp. 762-776, 2010.

[72] F. Macchetto, A. Marconi, D. J. Axon, A. Capetti, W. Sparks, and P. Crane, "The supermassive black hole of M87 and the kinematics of its associated gaseous disk," The Astrophysical Journal, vol. 489, no. 2, pp. 579-600, 1997.

[73] N. Nowak, R. P. Saglia, J. Thomas et al., "The supermassive black hole in NGC 4486a detected with SINFONI at the very large telescope," Monthly Notices of the Royal Astronomical Society, vol. 379, no. 3, pp. 909-914, 2007.

[74] J. Shen and K. Gebhardt, "The supermassive black hole and dark matter halo of NGC 4649 (M60)," The Astrophysical Journal, vol. 711, pp. 484-494, 2010. 
[75] G. de Francesco, A. Capetti, and A. Marconi, "Measuring supermassive black holes with gas kinematics. II. The LINERs IC 989, NGC 5077, and NGC 6500," Astronomy \& Astrophysics, vol. 479, pp. 355-363, 2008.

[76] N. Neumayer, M. Cappellari, J. Reunanen et al., "The central parsecs of centaurus A: high-excitation gas, a molecular disk, and the mass of the black hole," The Astrophysical Journal, vol. 671, no. 2, pp. 1329-1344, 2007.

[77] A. Capetti, A. Marconi, D. Macchetto, and D. Axon, "The supermassive black hole in the Seyfert 2 galaxy NGC 5252," Astronomy and Astrophysics, vol. 431, no. 2, pp. 465-475, 2005.

[78] L. Ferrarese and H. C. Ford, "Nuclear disks of gas and dust in early-type galaxies and the hunt for massive black holes: hubble Space Telescope observations of NGC 6251," The Astrophysical Journal, vol. 515, no. 2, pp. 583-602, 1999.

[79] R. P. van der Marel and F. C. van den Bosch, "Evidence for a $3 \times 108 \mathrm{M} \odot$ black hole in NGC 7052 from hubble space telescope observations of the nuclear gas disk," The Astronomical Journal, vol. 116, no. 5, pp. 2220-2236, 1998.

[80] M. Cappellari, E. K. Verolme, R. P. van der Marel et al., "The counterrotating core and the black hole mass of IC 1459," The Astrophysical Journal, vol. 578, no. 2, pp. 787-805, 2002.

[81] E. Dalla Bontà, L. Ferrarese, E. M. Corsini et al., "The highmass end of the black hole mass function: mass estimates in brightest cluster galaxies," The Astrophysical Journal, vol. 690, no. 1, pp. 537-559, 2009. 

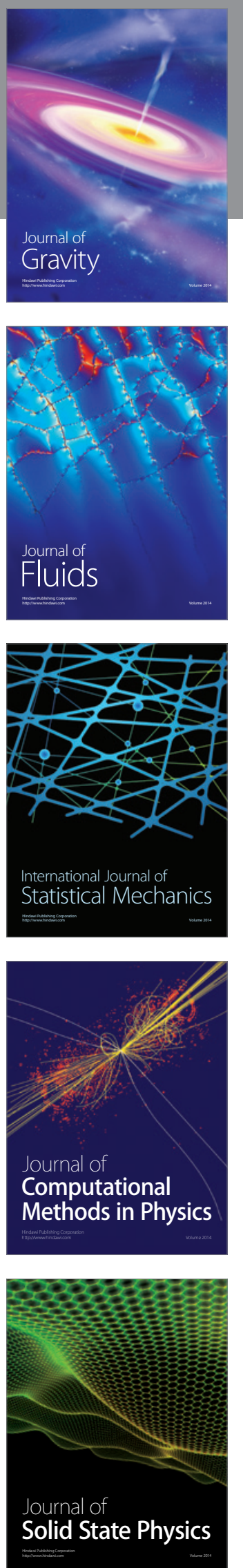

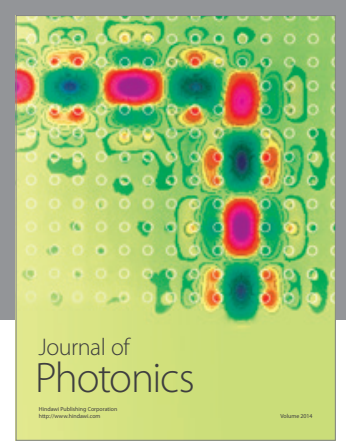

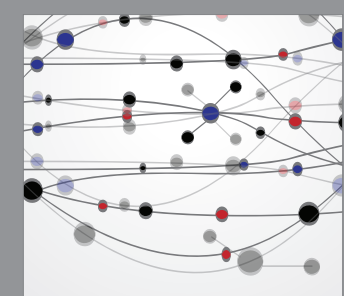

The Scientific World Journal
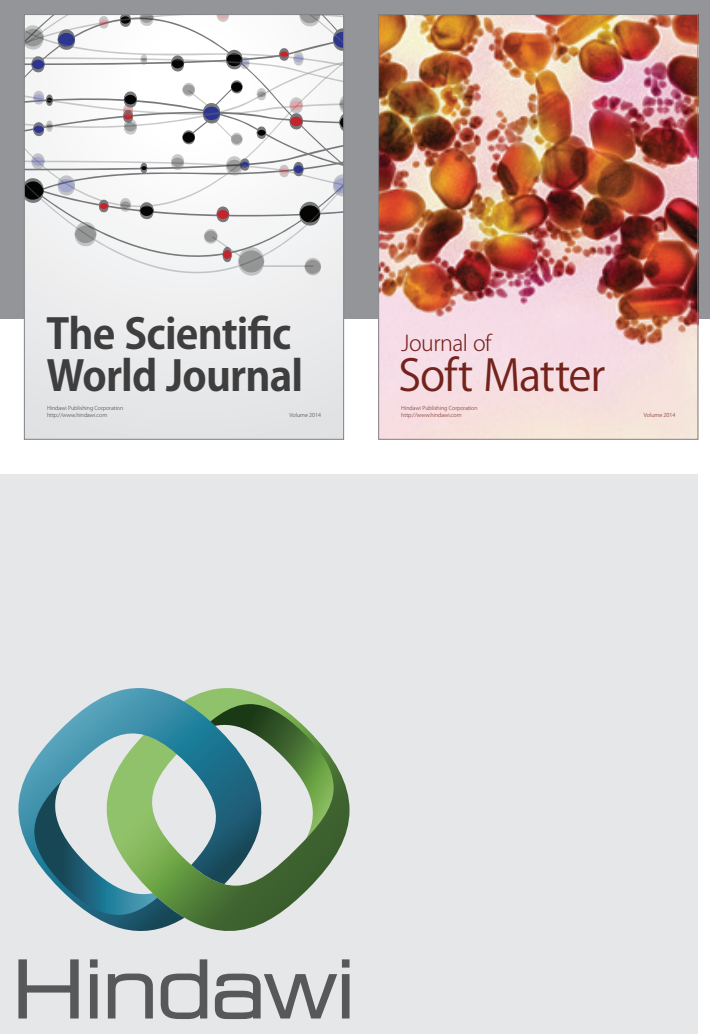

Submit your manuscripts at

http://www.hindawi.com
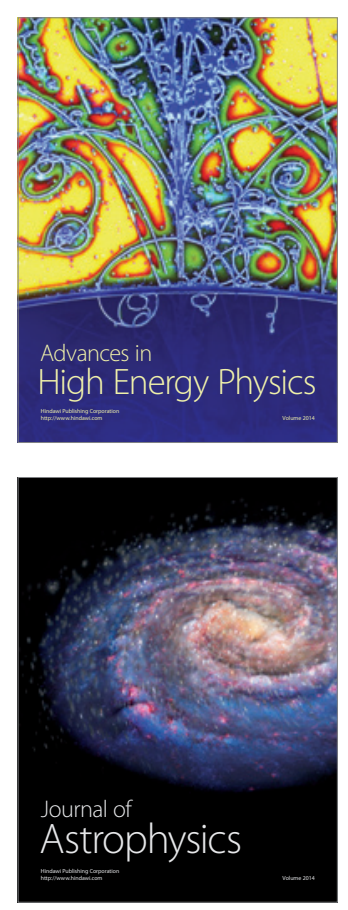
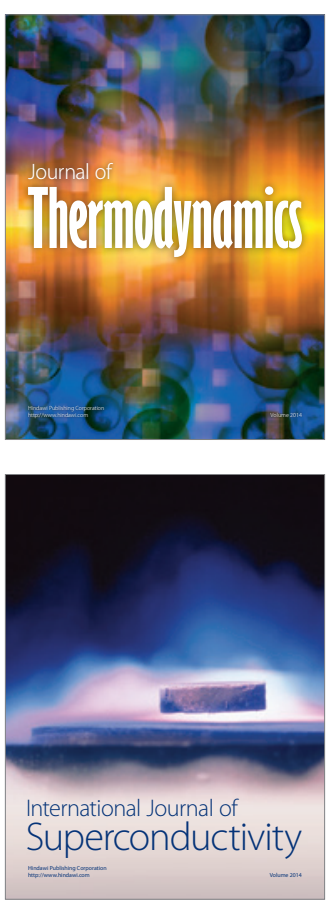
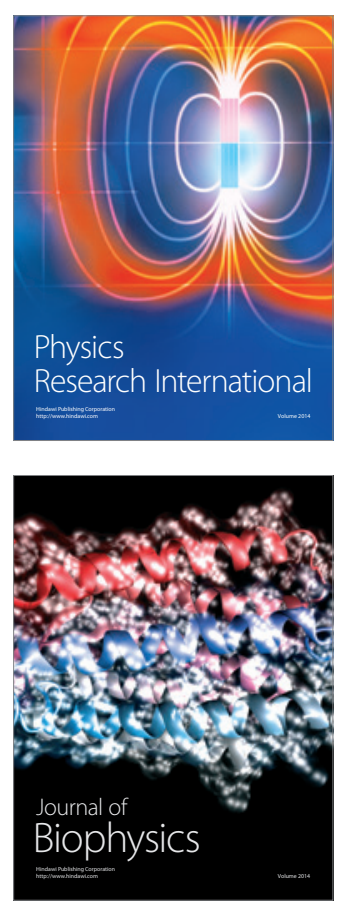
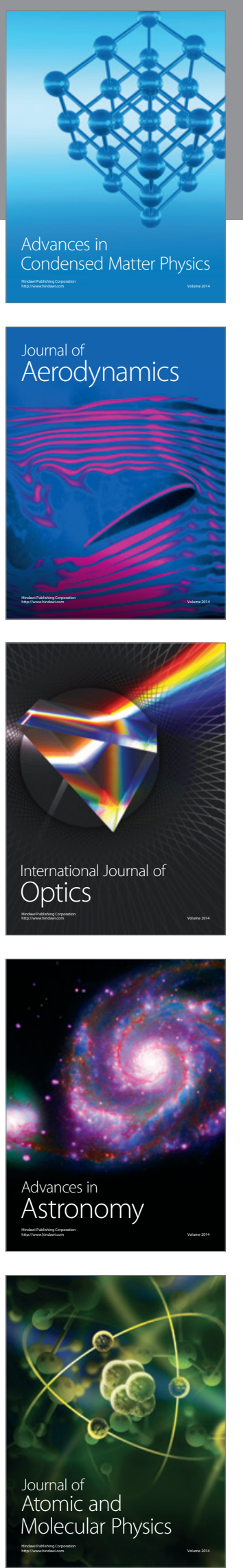